\title{
Diseño de tareas para promover aprendizaje autorregulado en la universidad
}

\section{Marcela Valencia-Serrano}

https://orcid.org/oooo-0001-7416-2393

Pontificia Universidad Javeriana,

Cali, Colombia

marcelav@javerianacali.edu.co

\section{Resumen}

El objetivo del artículo es proponer pautas para el diseño e implementación de tareas académicas que favorezcan el aprendizaje autorregulado en estudiantes universitarios. Las pautas toman en cuenta dos ejes: la estructura y la evaluación de la tarea. En cuanto a la estructura, se presentan pautas relacionadas con los objetivos, las consignas y el valor y promoción de la autonomía en la tarea para favorecer la autorregulación de los estudiantes. En la evaluación de la tarea se aborda el rol de los criterios de evaluación, la retroalimentación y los espacios de autoevaluación. Como conclusión se hace evidente la necesidad y conveniencia de la formación de los docentes en estrategias que favorezcan el aprendizaje autorregulado, a partir de las pautas propuestas en este trabajo, asi como de seguir demostrando empíricamente la efectividad de estas pautas en procesos especificos del aprendizaje autorregulado.

\section{Palabras clave (Fuente: tesauro de la Unesco)}

Aprendizaje autorregulado; estudiante universitario; formación de docentes; formación de profesores; pedagogía universitaria; práctica pedagógica.

Recepción: 27/08/2019 | Envío a pares: 22/01/2020 | Aceptación por pares: 30/03/2020 | Aprobación: 06/04/2020 


\title{
Task Design to Promote Self-Regulated Learning at University
}

\begin{abstract}
This article intends to propose guidelines for the design and implementation of academic tasks that favor self-regulated learning in university students. These guidelines consider two themes: task structure and assessment. The structure has to do with objectives, instructions, and the value and promotion of autonomy in the task to advance students' self-regulation. Task assessment addresses the role of evaluation criteria, feedback, and self-assessment opportunities. In conclusion, teacher training in strategies that promote self-regulated learning based on the proposed guidelines is necessary and convenient. Also, the effectiveness of the guidelines in specific selfregulated learning processes should be continuously and empirically demonstrated.
\end{abstract}

\section{Keywords (Source: Unesco thesaurus)}

Self-regulated learning; university students; teacher training; teacher education; university pedagogy; teaching practice. 


\section{Elaboração de tarefas para promover a aprendizagem autorregulada na universidade}

\section{Resumo}

Este artigo tem o objetivo de propor diretrizes para a elaboração e implementação de tarefas acadêmicas que favoreçam a aprendizagem autorregulada em estudantes universitários. Para isso, foram consideradas a estrutura e a avaliação da tarefa. Quanto à estrutura, são apresentadas diretrizes relacionadas com os objetivos, as instruções, o valor e a promoção da autonomia na tarefa para favorecer a autorregulação dos estudantes. Na avaliação da tarefa, o papel dos critérios de avaliação, a retroalimentação e os espaços de autoavaliação são abordados. Como conclusão, torna-se evidente a necessidade e conveniência da formação dos docentes em estratégias que favoreçam a aprendizagem autorregulada, a partir de diretrizes propostas neste trabalho, bem como da continuação efetiva dessas pautas em processos especificos da aprendizagem autorregulada.

\section{Palavras-chave (Fonte: tesauro da Unesco)}

Aprendizagem autorregulada; prática pedagógica; treinamento de professor; estudante universitário; pedagogia universitária. 
En la actual sociedad del conocimiento es imperativo que las personas no solo acumulen, sino que sepan cómo aprender nuevo conocimiento y cómo usarlo para responder a las dinámicas impuestas por la globalización, al auge de las tecnologías de la información y la comunicación y del mercado cambiante, de tal forma que puedan asegurar su bienestar personal, así como ejercer una ciudadanía responsable, que les permita contribuir al desarrollo social y económico (Severin, 2017). Lo anterior ha tenido un efecto en las metas formativas de todos los niveles educativos, pues estas se han enfocado en el desarrollo de competencias, para la formación de un un sujeto capaz de conocer y movilizar los conocimientos necesarios para la resolución de diversos problemas en contextos personales, académicos y laborales.

Binkley et al. (2012) agrupan las competencias para desarrollar en el siglo XXI en cuatro categorías: a) maneras de pensar: creatividad e innovación, pensamiento crítico, resolución de problemas y aprender a aprender; $b$ ) herramientas para trabajar: apropiación de tecnologías y manejo de información; c) maneras de trabajar: comunicación y colaboración; y d) maneras de vivir en el mundo: vida y carrera, responsabilidad personal y social, ciudadanía local y global.

Como consecuencia de las demandas de la sociedad del conocimiento y del énfasis puesto en el desarrollo de las competencias para el siglo XXI, se realizan nuevas demandas a las prácticas pedagógicas de los docentes, planteando que deben estar menos centradas en el profesor y más enfocadas en el aprendizaje y promoviendo espacios donde el estudiante tenga la posibilidad de descubrir la utilidad de los contenidos, aplicarlos y reflexionar sobre su uso idóneo en diferentes contextos (Chocarro et al., 2007; Ministerio de Educación Nacional, 2016).

Para Häkkinen et al. (2017) y Núñez et al. (2006), una de las competencias que más influye en la necesidad de transformar el currículo y las prácticas pedagógicas, centrándolas en el aprendizaje, es la competencia de aprender a aprender, definida como la forma de organizar y dirigir autónomamente el propio aprendizaje hacia el logro de metas (Binkley et al., 2012). Esto debido a que saber aprender es clave para el ajuste de los aprendices a los retos constantes que les plantea la universidad (Koivuniemi et al., 2017) y para la adaptación a las demandas que le hará el mercado laboral en el ejercicio de su profesión (Garello y Rinaduo, 2013).

Esta competencia de aprender a aprender ha sido abordada por la psicología educativa como aprendizaje autorregulado. La investigación sobre este concepto lleva más de 30 años y ha logrado probar la relación del concepto de regulación del aprendizaje con el desempeño académico de los estudiantes en diversas áreas y niveles educativos (Broadbent y Poon, 2015; Dent y Koenka, 2016; Schunk y Greene, 2017); así como ha comprobado la efectividad de diferentes estrategias aplicadas en contextos escolares y universitarios para que los estudiantes logren autorregularse (Boer et al., 2018; Donker et al., 2014; Bruijn et al., 2014).

Este texto tiene el objetivo de presentar un conjunto de pautas $u$ orientaciones dirigidas a profesores universitarios, con el fin de que estos puedan utilizarlos para el diseño de tareas académicas que logren fomentar en los estudiantes un aprendizaje autorregulado. Para esto, se inicia con una definición de aprendizaje autorregulado; seguidamente, se expone la importancia que tiene este constructo para la práctica pedagógica de los profesores; luego, se presentan las pautas para el diseño de tareas que fomenten aprendizaje autorregulado y se finaliza con algunas conclusiones.

\section{Aprendizaje autorregulado}

El aprendizaje autorregulado hace referencia al abordaje autónomo que hace un estudiante de una tarea o situación de aprendizaje (Hadwin, 2013). Esta autonomía se evidencia en el establecimiento de me- 
tas; en la planificación y despliegue de estrategias que hace el estudiante para lograr tales metas; en la supervisión de las estrategias y esfuerzos que despliega al resolverla; así como en la revisión que hace de su desempeño y aprendizaje en la tarea (Winne y Hadwin, 2008; Zimmerman, 2000; 2002; 2013). Este abordaje autónomo que hace el aprendiz también implica creencias motivacionales positivas -como la autoeficacia, el valor de tarea, el interés y la orientación a metas de aprendizaje-, la persistencia ante obstáculos que se puedan presentar y la voluntad de cumplir las demandas planteadas por las tareas (Zimmerman, 2011; Zimmerman y Moylan, 2009).

Autores como Winne (2018) y Winne y Hadwin $(1998 ; 2008)$ plantean que el aprendizaje autorregulado ocurre en cuatro fases recursivas:

- Fase I: Definición de la tarea, donde el estudiante identifica cuáles son las condiciones internas (conocimientos previos que posee sobre contenidos y estrategias para cumplir las demandas de la tarea, estado emocional ante la tarea, autoeficacia, intereses, expectativas, etc.) y condiciones externas de la tarea que debe realizar (ambiente, tiempo, instrucciones, demandas de la tarea). La identificación de estas condiciones le permite al aprendiz construir una representación de la tarea: sus retos, nivel de dificultad y posibilidades de alcanzarla (Greene y Azevedo, 2007; Winne y Hadwin, 1998, 2008)

- Fase 2: Establecimiento de metas y planificación: fase en la que el estudiante, con base en la representación que construyó de la tarea en la fase anterior, procede a establecer metas de aprendizaje o estándares y a elaborar un plan que le permita alcanzar dichas metas. Este plan se compone de: a) pasos para alcanzar los estándares: desde aspectos superficiales como el tiempo a dedicar, el espacio y los materiales a usar, hasta aspectos profundos, como las estrategias y tácticas de aprendizaje que nece- sita para abordar la tarea; y b) los productos a lograr derivados de las actividades en la tarea (Winne, 2017).

- $\quad$ Fase III: Implementación de estrategias y tácticas cognitivas y adaptaciones sobre la marcha, donde el aprendiz realiza dos actividades simultáneamente (Winne, 2017): la primera es la ejecución del plan propuesto en la Fase 2, implementando estrategias y tácticas cognitivas pertinentes para la resolución de la tarea, como las estrategias de selección y la táctica del subrayado, entre otras operaciones cognitivas. La segunda actividad es de carácter metacognitivo e implica el despliegue de monitoreo para establecer qué tan efectivas están siendo las estrategias planeadas para lograr la tarea y manejar tanto el ritmo como los esfuerzos que se están realizando. A partir de este monitoreo, se generan juicios que pueden derivar en la realización de control metacognitivo, para cambiar estrategias, condiciones internas, externas o el plan (Winne, 2011; 2018).

- $\quad$ Fase IV: Adaptación metacognitiva, que ocurre al final de la ejecución, cuando el estudiante hace una revisión de todas las operaciones y productos generados en las fases anteriores durante el abordaje de la tarea (Winne y Hadwin, 1998; Winne, 2018). A partir de esa revisión, el estudiante genera una reflexión sobre su aprendizaje y desempeño en la tarea, así como sus logros y fallos en ella, y logra derivar posibles cambios a realizar en el abordaje de futuras tareas. Estos cambios pueden darse, según Winne y Hadwin (2008) en las condiciones internas (consolidar más conocimiento de un dominio), en los estándares (el aprendiz puede caer en cuenta de que requiere exigirse más para una tarea posterior) y en las operaciones o estrategias (después de ejecutar una tarea, el aprendiz percibe que para ese tipo de actividad tiene que desplegar ciertas estrategias y evitar otras). 
Un estudiante autorregulado puede establecer metas orientadas al aprendizaje, sostener el interés por lo que hace y persistir en ello; escoger estrategias acordes con las demandas de la tarea; monitorear constantemente la efectividad de esas estrategias; realizar ajustes a ellas, si lo requiere; $y$ reflexionar sobre su desempeño global al final de las tareas (Rosario et al., 2014; Torrano et al., 2017; Winne, 2011; Zimmerman, 2002; 2013). Estos estudiantes suelen consolidar aprendizajes de calidad y presentar altos desempeños académicos (Abar y Loken, 2010; Tuysuzoglu y Greene, 2015; Broadbent y Poon, 2015; Daura, 2015; Greene et al., 2012; 2015; Kitsantas, 2002; McCardle y Hadwin, 2015; Montes et al., 2005; Torrano et al., 2017; Valencia et al., 2013).

Las características descritas definen el funcionamiento ideal de los estudiantes en cuanto a la toma de control y autonomía en su aprendizaje en el aula de clase y fuera de esta. Este funcionamiento es susceptible de ser aprendido y refinado gracias a las interacciones del alumno en múltiples contextos (Ben y Bernacki, 2015). Específicamente, en un contexto formal de educación, las interacciones con profesores y pares y las actividades académicas que suceden en este contexto contribuyen a la formación de las habilidades de autorregulación del estudiante (Daura, 2011; 2013; Hadwin et al., 2017; Lajoie, 2008).

Es así como el diseño de tareas académicas significativas y el andamiaje que el docente dé al estudiante para apoyarlo en la ejecución de las tareas, son fundamentales para ejercer regulación externa sobre el proceso de aprendizaje del estudiante y entregarle gradualmente el control sobre su proceso de aprendizaje (Hadwin et al., 2005; Hadwin et al., 2011; 2017; Paris y Paris, 2001).

El apoyo externo posibilita que el estudiante empiece a consolidar los procesos cognitivos, metacognitivos y motivacionales involucrados en el aprendizaje autorregulado y que los despliegue, en pro de lograr los objetivos de aprendizaje que se le plantean (Hadwin et al., 2017; Winne y Buttler,
1995; Zimmerman, 2002). Con el avance en los grados escolares, se observa que los estudiantes toman más control de su aprendizaje, dependiendo menos del control externo y siendo capaces de elaborar sus propias metas de aprendizaje, de elegir estrategias a utilizar y cuándo ajustarlas, de ser más reflexivos sobre su propio aprendizaje y de tener más herramientas para persistir ante dificultades (Dent y Koenka, 2016).

Sin embargo, esto no quiere decir que se deba retirar el apoyo al proceso de autorregulación o que los estudiantes en niveles avanzados de educación, como el universitario, logren ser autorregulados. Respecto a esto, la evidencia empírica ha mostrado que los estudiantes universitarios son expuestos a múltiples retos cognitivos, emocionales y motivacionales en el contexto universitario ligados a las actividades académicas y no académicas, a los cuales estos deben ajustarse para lograr aprendizajes y buenos desempeños (Koivuniemi et al., 2017).

En ese proceso de ajuste, los estudiantes presentan algunas dificultades para autorregular su aprendizaje, que perjudican su desempeño, dificultades como: a) uso de estrategias inadecuadas para lograr las demandas de la tarea; b) dificultades en definir la tarea, poca planeación y bajo monitoreo de su desempeño durante las tareas; c) discrepancias fuertes entre su desempeño real y su desempeño percibido; d) bajo valor de las tareas y contenidos y baja autoeficacia; e) poca búsqueda de ayuda; y f) control inadecuado de distracciones, ansiedad y baja persistencia (Tuysuzogluy Greene, 2015; Capote et al., 2017; Greene et al., 2012; 2015; Lawanto y Febrian, 2017; Montes et al., 2005; Nelson et al., 2015; Shell y Soh, 2013; Valencia et al., 2013).

Las dificultades mencionadas en el proceso de autorregulación de los estudiantes y la importancia de fomentar ese proceso para su formación profesional imponen retos importantes a los profesores universitarios, puesto que implica para ellos implementar prácticas pedagógicas orientadas a apoyar a 
los estudiantes, no solo en el aprendizaje de los contenidos, sino en: a) la identificación y comprensión de los retos que enfrentan en el aprendizaje de los contenidos; b) la elección de acciones propicias para ajustarse a esos retos; y c) la apropiación de conocimientos y estrategias que les ayuden a planear, a monitorear y a reflexionar sobre su propio proceso de aprendizaje en la universidad y, más adelante, en su ejercicio profesional.

\section{Importancia del aprendizaje autorregulado para la práctica pedagógica de los profesores}

Comprender el constructo del aprendizaje autorregulado se constituye en un aspecto clave para que los profesores puedan enfrentar los desafíos mencionados y transformar su práctica pedagógica, con el fin de favorecer la regulación del aprendizaje en sus estudiantes. A este respecto, autores como Dignath (2016), Dignath y Büttner (2018), Dignath et al. (2013), Dignath y Van der Werf (2012), Kistner et al. (2015) plantean que, cuando los docentes tienen conocimientos sobre el aprendizaje autorregulado y creen que este es importante para potenciar el desempeño y el aprendizaje de los estudiantes, es más probable que promuevan estrategias explícitas de autorregulación en sus clases, como la organización y la planeación, y generen ambientes de clase basados en el constructivismo (promoción de la autonomía, tareas desafiantes y ancladas al contexto real y trabajo colaborativo), los cuales favorecen en los estudiantes el uso de estrategias de autorregulación.

Por su parte, Paris y Paris (2001) plantean que, cuando los profesores están familiarizados con el aprendizaje autorregulado, es más probable que estos sean más organizados y reflexivos sobre su propia práctica pedagógica y que logren regular su enseñanza, planificándola, organizándola y reflexionando sobre sus aciertos y desaciertos en el aula. El ser reflexivos sobre su propia forma de enseñar ayuda a que los profesores se vuelvan más sensibles y logren comprender mejor las estrategias que los estudiantes usan para aprender y monitorear su aprendizaje, lo que los ayudaría a estructurar una práctica pedagógica que guíe a sus estudiantes hacia formas efectivas de regular su aprendizaje en las tareas, con lo que se convertirían en modelos idóneos para estos en el proceso de aprender a aprender (Paris y Winograd, 2003; Peeters et al., 2014).

Häkkinen et al. (2017), Kramarski (2017) y Perry (1998) proponen que, para lograr que los profesores propicien en sus estudiantes aprendizaje autorregulado, es necesario que durante la formación de los docentes en las licenciaturas se impartan asignaturas donde se aborde el tema de la regulación del aprendizaje. Adicionalmente, la formación de los profesores no debe agotarse en las licenciaturas, sino que también se debe trabajar con los profesores en servicio, ofreciendo programas de formación y actualización que aborden el tema de la autorregulación o promoviendo comunidades de aprendizaje entre los profesores donde, guiados por mentores, puedan reflexionar sobre su propia enseñanza y sobre las formas de promover la autorregulación en sus estudiantes (Buttler et al., 2013; Kramarski y Cohen, 2016; Kramarski et al., 2013; Rosario et al., 2014).

La evidencia empírica ha comprobado que, en la medida en que se instruya a los profesores en formas de autorregular su enseñanza y en estrategias puntuales para promover el aprendizaje autorregulado en sus estudiantes (como tutorías, retroalimentación entre pares, discusión de objetivos y productos de la tarea, instrucción en planeación), mejorarán sustancialmente las habilidades de autorregulación de los estudiantes, como la planeación, el monitoreo y el control (De la Fuente et al., 2013; Kramarski, 2017; Kramarsky y Michalsky, 2010; Perry et al., 2008; Perry et al., 2006; Vrieling et al., 2012).

Respecto a la formación de profesores universitarios para promover aprendizaje autorregulado, Moos y Ringdal (2012) proponen fortalecer los programas de formación en estos profesores, ya que po- 
seen gran dominio de su área de conocimiento, pero dificultades en su formación pedagógica, lo que hace que al enseñar se centren más en la transmisión de contenidos y poco en reflexionar con sus estudiantes sobre el proceso de aprendizaje. En cuanto a esto, Daura (2011; 2013) y Hoops et al. (2016) encontraron, al observar clases de profesores universitarios, que estos se centraban más en la exposición magistral de conceptos y poco en la generación de actividades donde los estudiantes pudieran reflexionar sobre sus dificultades para comprender los conceptos y sobre las formas de aprenderlos idóneamente.

Resultados similares fueron hallados por Valencia y Caicedo (2017), quienes, al analizar tareas académicas propuestas por docentes universitarios, observaron que muy pocas tenían el potencial para favorecer un aprendizaje autorregulado; por ejemplo, un grupo de esas tareas exigió altos esfuerzos cognitivos por parte de los estudiantes, pero hubo poco acompañamiento por parte del profesor para guiar al estudiante hacia el logro de la tarea.

\section{Pautas para el diseño de tareas que favorezcan aprendices autorregulados}

Las pautas para promover aprendizaje autorregulado se presentan tomando en cuenta dos ejes: la estructura de la tarea y la evaluación de la tarea. En cada uno de estos ejes se dan sugerencias para diseñar las tareas, de tal forma que ayuden a promover el despliegue idóneo de las diferentes fases del aprendizaje autorregulado en los aprendices. Estas pautas se basan en planteamientos teóricos y estudios empíricos realizados por autores de referencia en el tema del aprendizaje autorregulado, publicados entre 1998 y 2019, enfocados en estudiar el aprendizaje autorregulado en relación con aspectos instruccionales, tales como: la promoción de autonomía, la evaluación y retroalimentación y el planteamiento de tareas desafiantes. La búsqueda se concentró en escenarios universitarios, aunque algunos de los artículos revisados, especialmente los relacionados con la estructura de la tarea, per- tenecen a autores de referencia en el tema, cuyas reflexiones y hallazgos se concentran en escenarios de primaria y secundaria (ejemplo: Nancy Perry) y son pertinentes para pensar el diseño de tareas en la educación superior.

\section{Estructura de la tarea}

Al diseñar una tarea académica se deben tener en cuenta ciertos elementos cruciales, que determinan la comprensión de esta por parte de los estudiantes. Elementos como: el tipo de tarea, las demandas de esta, los objetivos, las instrucciones que se dan al estudiante para resolverla (Häkkinen et al., 2017; Lodewyk et al., 2009; Orozco et al., 2002) y la promoción de la autonomía en la tarea (León et al., 2015; Perry et al., 2018; Wang et al., 2016).

1. TIPO DE TAREA: PLANTEAR TAREAS ABIERTAS Y DESAFIANTES $Y$ QUE SE EXTIENDAN EN EL TIEMPO

Las tareas académicas deben ser abiertas: implicar más de una solución, permitir diferentes caminos para su logro y extenderse en el tiempo, es decir, que su resolución implique más de un intento por parte de los estudiantes. Asimismo, las tareas deben plantear demandas cognitivas de alto orden (aplicar, argumentar, crear, entre otras), que activen el uso de conocimientos previos y el anclaje de estos a nuevos contenidos. Ejemplos de tareas abiertas, demandantes y extendidas en el tiempo son: la resolución de problemas reales, a partir de la generación de propuestas de intervención o de productos tangibles, como dispositivos electrónicos; la realización de diagnósticos; el análisis de casos; la creación y ejecución de proyectos; la elaboración de ensayos, entre otras.

Este tipo de tareas exigen a los estudiantes plantearse metas y organizar sus acciones para alcanzarlas (Fase II: Establecimiento de metas y planificación). Además, promueven el uso de estrategias de procesamiento profundo, como elaborar, organizar, personalizar y transferir conocimiento (Häkkinen et al., 2017; McMahon y Oliver, 2001; Perry, 1998; 
Perry et al., 2018). En lo motivacional, el desafío que provocan las altas demandas despierta el interés por la tarea, lo que ayuda al despliegue de los esfuerzos cognitivos e influye en que los estudiantes persistan en la tarea durante su ejecución (Fase III) (Puntel y Menna, 2018; Perry et al., 2002; Lodewyk et al., 2009).

2. Plantear tareas oue impliouen el conocimiento previo DE LOS ESTUDIANTES

Las tareas deben estar ancladas a los conocimientos previos de los estudiantes y procurar la integración de estos con los nuevos contenidos de aprendizaje, dándole la oportunidad al estudiante de ajustarse a las demandas de la tarea y construir nuevo conocimiento. Por ejemplo, en una asignatura de psicología se propone una tarea en la que se debe crear un programa psicoeducativo donde es necesario que los estudiantes activen conocimientos previos relacionados con las teorías del desarrollo y el aprendizaje que se abordaron en semestre anteriores y que los integren con contenidos abordados en clase sobre cómo diseñar un programa de intervención psicoeducativo (Valencia y Caicedo, 2017).

Al retomar los conocimientos previos de los estudiantes y promover su anclaje con nuevos contenidos, se está contribuyendo a que el estudiante tenga un punto de partida para iniciar el establecimiento de metas y la estructuración de un plan para lograrlas (Fase II), de tal forma que pueda determinar qué sabe sobre la tarea y qué necesita conocer o fortalecer para lograrla (Greene y Azevedo, 2007; Winne, 2018; Winne y Hadwin, 2008). Igualmente, al tener en cuenta los conocimientos previos, el estudiante podrá supervisar y evaluar el nivel transformación que estos experimentan durante la resolución de la tarea y al final de esta (Garello y Rinaudo, 2012).

3. Plantear tareas oue estén contextualizadas o sean AUTÉNTICAS

Procurar que las tareas tengan relación con el contexto real donde están los estudiantes, para que así estos puedan ver la relevancia de los contenidos que aprenden, para comprender y resolver problemáticas de la realidad y para su formación profesional. Por ejemplo, el diseño del programa psicoeducativo debe hacerse tomando como punto de referencia una población, los estudiantes deben contactar a las personas y realizar un ejercicio diagnóstico con ellas, con el fin de identificar una necesidad educativa que fundamente el diseño del programa.

Cuando una tarea está contextualizada y es relevante, impacta la motivación de los estudiantes, activando creencias relacionadas con el valor o utilidad de la tarea y el interés en la misma. Este impacto motivacional es importante, en la medida en que puede llevar a que los estudiantes se dispongan a realizar y persistir en esfuerzos cognitivos (Fase III), que los dirijan al logro de las situaciones reales que les plantea la tarea (Dignath et al., 2013; McMahon y Oliver, 2001; Perry y Rahim, 2011; Perry et al., 2004).

\section{Plantear consignas claras para la tarea académica}

Las consignas deben explicitar con claridad: a) los objetivos de la tarea en términos de aprendizaje; b) el producto o los productos a lograr a partir de la ejecución de la tarea; c) los pasos o instrucciones guía para alcanzar los objetivos y productos; y d) tiempos y plazos para las entregas de los productos. Estos elementos de la consigna pueden estar plasmados en un medio escrito, en forma de guía, que puede ser entregada en físico o presentada en un medio virtual, para que el estudiante tenga acceso a ella de forma permanente. Además de esto, para mejorar la comprensión de la tarea por los estudiantes, es importante que el profesor explique las instrucciones, esté atento a resolver posibles dudas y cuente con modelos exitosos de productos, que puedan servirles de guía para representar lo que se les está pidiendo (Hawe y Dixon, 2017).

Estas recomendaciones en cuanto a la consigna contribuyen a facilitar las operaciones que se llevan a cabo en las Fases I y II: Definición de la tarea 
y Establecimiento de metas y planificación, en la medida en que le permiten al estudiante alcanzar una comprensión general de la tarea: demandas de la misma, producto, recursos con los que cuenta, posibilidades de alcanzarla; así, con base en esto, podrá generar estándares y pasos para lograr la tarea, decidiendo qué estrategias usar, qué tiempo destinar y qué recursos buscar (Careless, 2016; Hawe y Dixon, 2017; Ley y Young, 2001; Lodewyk et al., 2009; Winne y Jamieson, 2002).

\section{PROMOCIÓN DE LA AUTONOMía}

Es relevante considerar la participación y grado de control de los estudiantes en la tarea académica. Esta participación consiste en permitir que decidan formas de estructurar y ejecutarla, para que sientan que tienen control en la actividad. En este sentido, puede dárseles la oportunidad de escoger el nivel de reto a emprender, las formas de presentación de estos contenidos, materiales a utilizar y/o productos a lograr. Para ilustrar: en una tarea sobre comunicación persuasiva, estudiada en la investigación de Valencia y Caicedo (2017), el docente permite a sus alumnos decidir el tipo de producto que generarán (material visual como pósters, material audiovisual como videos, entre otros) y escoger la población a la que dirigirán el producto (niños, adultos, ancianos).

Las opciones en la toma de decisiones son importantes para facilitar el aprendizaje autorregulado, en la medida en que brindan al estudiante la oportunidad de tomar control sobre su desempeño en la tarea y sentirse responsables de su aprendizaje, lo cual les ayuda a activar aspectos motivacionales claves para resolverla (Fase I: Definición de la tarea) -como el interés en la tarea y la creencias de autoeficacia positivas-, al sentir que pueden con el reto planteado. Por ello son indispensables para activar los procesos de comprensión, aplicación y monitoreo necesarios para generar aprendizajes en la tarea y persistir en esta (Fase III) (León et al., 2015; Perry et al., 2018; Perry y Rahim, 2011; Perry y VanderKamp, 2000; Perry et al., 2002).

\section{Evaluación de la tarea centrada en el aprendizaje}

Otro eje, fundamental para pensar el diseño y ejecución de las tareas y fomentar aprendizaje autorregulado en los estudiantes, es la evaluación que se hará de su desempeño en la tarea. Esta evaluación debe tener carácter formativo y se caracteriza por fomentar que el estudiante comprenda profundamente los estándares que debe lograr y los criterios que se utilizarán para evaluar su desempeño. Asimismo, provee información pertinente al estudiante sobre el estado de su aprendizaje y desempeño durante y al final de la ejecución y propone alternativas para que mejore sus aprendizajes y formas de abordar la tarea (Panadero et al., 2019; William, 2014).

6. Generar criterios de eValuación claros para los proDUCTOS DE LA TAREA

Los estudiantes deben conocer los criterios que se tendrán en cuenta para evaluar su desempeño en la tarea, de tal forma que puedan saber cuál es el desempeño esperado y los estándares con los que será evaluado ese desempeño. Estos criterios pueden ser especificados al inicio de la tarea y discutidos con los estudiantes, para que puedan comprenderlos. Ejemplo, en el diseño de un programa psicoeducativo, el profesor socializa y explica a los estudiantes cuatro criterios para evaluar el diseño: a) pertinencia de la propuesta para solventar la necesidad de la población; b) coherencia de la propuesta con los planteamientos teóricos abordados en clase; c) coherencia de los objetivos de la intervención con las actividades planteadas; y d) actividad para evaluar la efectividad de la propuesta

Según Fraile et al. (2017a) y Panadero y Alonso (2013), estos criterios pueden hacerse operativos en rúbricas de evaluación que describan diferentes niveles de desempeño (bajo, medio, alto; novato, intermedio, experto); también, pueden ser descritos en forma de preguntas o guiones (¿qué tanto la actividad 1 es coherente con el objetivo 1?), que le ayuden al estudiante a pensar en qué medida sus avances están logrando responder esas cuestiones a cabalidad. 
Explicar los criterios de evaluación a los estudiantes es una actividad que facilita varios procesos involucrados en la autorregulación del aprendizaje (Tabla 1).

Por último, en relación con el uso de rúbricas o guiones para explicitar los criterios de evaluación, algunos estudios sugieren que la escogencia de una u otra herramienta debe realizarse tomando en cuenta las características de la tarea y el nivel de experticia de los estudiantes en ella (Panadero y Jonsson, 2013; Panadero et al., 2014; 2013). De este modo, las rúbricas han demostrado ser más efectivas en tareas que se van a calificar y en las que el aprendiz tiene poca experiencia o son novedosas para él, pues lo ayudan a disminuir pensamientos negativos sobre su desempeño y la ansiedad al ejecutar la tarea, mientras que los guiones parecen ser más adecuados en tareas complejas, en las cuales el estudiante tendría un poco de más experticia, al promover en él el aumento de esfuerzos, de mensajes autodirigidos durante el desempeño y el uso de diferentes estrategias de aprendizaje.

\section{DAR RETROAlimentación A LOS AVANCES EN LA TAREA}

Una retroalimentación efectiva para propiciar el aprendizaje autorregulado puede tener en cuenta los siguientes aspectos:

1. Las recomendaciones y observaciones a los estudiantes deben ser coherentes con lo exigido por la tarea y con sus criterios de evaluación, de tal forma que se impulsen en ellos procesos de autoevaluación de su propio desempeño en la tarea (Tai et al., 2018). Igualmente, deben ser acordes al proceso de los alumnos en la tarea, para no generar confusión y perjudicar su desempeño. Para esto, Careless (2016) propone que, para generar sus observaciones y sugerencias, el docente revise exhaustivamente los productos preliminares del estudiante y dialogue con él sobre: a) cómo percibe su proceso en la tarea; b) qué piensa de los productos que hasta ahora ha generado; y c) qué considera qué ha logrado y en qué debe mejorar. Además, la retroalimentación debe ser proactiva, apoyando a los estu-

Tabla 1. Aportes del establecimiento de criterios de evaluación

al aprendizaje autorregulado

\begin{tabular}{|c|c|c|}
\hline $\begin{array}{c}\text { Fase I y Fase II: Definición de la } \\
\text { tarea y Establecimiento de metas y } \\
\text { planificación }\end{array}$ & $\begin{array}{l}\text { Fase III: Ejecución de la tarea y } \\
\text { ajustes sobre la marcha }\end{array}$ & Fase IV: adaptación metacognitiva \\
\hline $\begin{array}{l}\text { Facilitan a los estudiantes la creación } \\
\text { de estándares y la planeación de } \\
\text { sus acciones para lograrlos. La } \\
\text { creación de estándares también } \\
\text { les permite escoger estrategias } \\
\text { cognitivas acordes para alcanzar } \\
\text { dichos estándares (Nicol, 2009; Nicol } \\
\text { y MacFarlane-Dick, 2006; Panadero y } \\
\text { Jonsson, 2013; Panadero et al., 2016). }\end{array}$ & $\begin{array}{l}\text { Facilita el monitoreo de la } \\
\text { efectividad de las estrategias } \\
\text { cognitivas usadas para lograr } \\
\text { la tarea. El monitoreo permite a } \\
\text { los estudiantes generar juicios } \\
\text { evaluativos sobre qué tanto están } \\
\text { avanzando o fallando en la ejecución } \\
\text { de la tarea, juicios que motivarán la } \\
\text { realización de acciones para lograr } \\
\text { cumplir las demandas de la tarea: } \\
\text { control metacognitivo (Tai et al., } \\
\text { 2018; Winne y Buttler, 1995). }\end{array}$ & $\begin{array}{l}\text { Apoya la reflexión final del } \\
\text { estudiante sobre su desempeño } \\
\text { global en la tarea: qué tanto logró } \\
\text { los estándares, qué tanto aprendió, } \\
\text { qué fallos tuvo. Con base en ello, } \\
\text { podrá pensar cómo lo aprendido en } \\
\text { esa tarea le ayuda en otras y cómo } \\
\text { los fallos pueden ser mejorados en } \\
\text { futuras asignaciones (Hawe y Dixon, } \\
\text { 2017; Nicol, 20o9; Nicol y MacFarlane- } \\
\text { Dick, 2006; Panadero et al., 2016). }\end{array}$ \\
\hline
\end{tabular}

Fuente: Elaboración propia. 
diantes, no solo en la identificación de sus fallos en la tarea, sino también en la generación de acciones para solventar esos fallos y seguir avanzando en el ejercicio (Hattie y Temperley, 2007; 2011; Careless y Boud, 2018).

2. La retroalimentación brindada a los estudiantes debe tener en cuenta aspectos motivacionales y emocionales. Si se percibe que están frustrados, ansiosos, desconfían de sus habilidades o han perdido el sentido de la tarea, es importante que el profesor genere un ambiente de confianza y dialogue con ellos al respecto, para que puedan determinar la fuente de estos estados y generen estrategias para disminuirlos (Careless, 2013; Nicol y MacFarlane, 2006; Rojas y Valencia, en revisión). Ejemplo: para disminuir la ansiedad, la desconfianza y la frustración es productivo señalar a los estudiantes los puntos fuertes que se observan en el abordaje que hacen de la tarea, explicarles detalladamente por qué están fallando, normalizar los fallos, es decir, indicarles que pueden suceder, y mostrarles que estos tienen solución.

3. Involucrar a los pares en la retroalimentación, generando espacios para que emitan juicios sobre los avances de sus compañeros en la tarea. Esta retroalimentación puede ser guiada por criterios de evaluación de la tarea, generados por el profesor o coconstruidos con los estudiantes (Fraile et al., 2017b). De igual forma, el profesor puede decidir que los estudiantes que mostraron avances significativos en las tareas sean los que retroalimenten a los que están presentando dificultades. Los pares, al poseer una percepción más cercana de los retos y dificultades de la tarea, pueden hacer juicios más comprensibles para sus compañeros y ofrecer formas de mejorar beneficiosas para el abordaje que estos hacen de la tarea (Hawey Dixon, 2017; Nicol y MacFarlane, 2006; Panadero y Alonso, 2013; Panadero, Jonsson et al., 2018; Panadero et al., 2016; To y Panadero, 2019).
Es preciso mencionar que las anteriores recomendaciones sobre la retroalimentación son pertinentes para facilitar el aprendizaje autorregulado de los estudiantes, en la medida en que permiten que estos cuenten con una fuente externa de información que complemente las comparaciones que ellos hacen entre sus avances en la tarea y los estándares que establecieron en la Fase II (Winne y Buttler, 1995). La Tabla 2 explicita los aportes más importantes de la retroalimentación al proceso de autorregulación del aprendizaje.

8. Generar espacios para oue el estudiante pueda aUtoeVALUARSE DURANTE Y DESPUÉS DE LA TAREA

Los espacios de autoevaluación son importantes para que el estudiante pueda reflexionar sobre su desempeño y sus resultados en la tarea y plantear formas de seguir mejorando. Para guiarlo en la autoevaluaión de su desempeño y aprendizaje, son cruciales los criterios, en forma de rúbricas o guiones, pues, como se mencionó, ayudan a los estudiantes a generar estándares frente a los cuales comparar su desempeño en la tarea, lo cual fortalece el monitoreo metacognitivo o la elaboración de juicios sobre su abordaje en la ejecución de la tarea (Panadero et al., 2013; 2014; Tai et al., 2018).

Es importante que el profesor estructure la situación para que el estudiante emita sus propios juicios evaluativos. Por ejemplo: si el espacio de autoevaluación se genera durante la ejecución de la tarea, hay que indicarle: a) qué debe evaluar de su proceso: el producto preliminar logrado hasta el momento, los procesos realizados para llegar a ese producto; b) cómo hacerlo: usando la rúbrica o el guion creados para tal fin, respondiendo preguntas reflexivas dadas por el profesor; c) en qué medio hacerlo: oralmente y grabándose, o por escrito, usando un diario o portafolio, entre otros. Una vez el estudiante ha generado sus juicios, es importante que el profesor lo escuche y que, como ya se mencionó, dé retroalimentación al respecto, para refinar esos juicios y dialogar sobre las formas de mejorar el desempeño. 
Tabla 2. Aportes de la retroalimentación al aprendizaje autorregulado

\begin{tabular}{|l|l|}
\hline \multicolumn{1}{|c|}{$\begin{array}{c}\text { Apoyo de la retroalimentación en la tarea al aprendizaje } \\
\text { autorregulado de los estudiantes }\end{array}$} & \multicolumn{1}{c|}{ Ejemplo } \\
\hline $\begin{array}{l}\text { En la Fase III, la retroalimentación optimiza el monitoreo } \\
\text { y los juicios evaluativos que hace el estudiante durante la } \\
\text { ejecución de la tarea (Adie et al., 2018; Hawe y Dixon, 2017; } \\
\text { Nicol y MacFarlane, 2006; Tai et al., 2018; Winne y Buttler, } \\
\text { 1995). }\end{array}$ & $\begin{array}{l}\text { Si el estudiante está subvalorando sus avances en } \\
\text { la tarea, omitiendo los aspectos positivos de su } \\
\text { desempeño, entonces la retroalimentación será } \\
\text { de utilidad para demostrarle que hay avances } \\
\text { significativos que lo acercan al estándar. }\end{array}$ \\
\hline $\begin{array}{l}\text { La retroalimentación facilita que los aprendices fortalezcan } \\
\text { los procesos de control metacognitivo (Fase III), lo que } \\
\text { llevará a la realización de ajustes en el abordaje de la tarea, } \\
\text { cambiando estrategias de aprendizaje, modificando el plan, } \\
\text { entre otros (Hattie y Gan, 2011; Nicol, 20og; Tai et al., 2018; } \\
\text { Winne y Buttler, 1995). }\end{array}$ & $\begin{array}{l}\text { A partir de la retroalimentación, un estudiante } \\
\text { puede decidir cambiar la forma en la que ha estado } \\
\text { resolviendo la tarea: pasar de una estrategia de } \\
\text { repetición a una de elaboración. }\end{array}$ \\
$\begin{array}{l}\text { Mejora la motivación de los estudiantes y eleva sus esfuerzos, } \\
\text { su interés por la tarea y la persistencia en la misma, al } \\
\text { mostrarles que sí es posible mejorar y lograr las demandas } \\
\text { del trabajo (Careless, 2016; Rojas y Valencia (en revisión). }\end{array}$ & $\begin{array}{l}\text { El estudiante que cambia su estrategias de } \\
\text { repetición por una estrategia de elaboración } \\
\text { persiste en la ejecución de la tarea y, a su vez, eleva } \\
\text { su interés y esfuerzos, al ver que la nueva estrategia } \\
\text { funciona mejor que la anterior. }\end{array}$ \\
\hline
\end{tabular}

Fuente: Elaboración propia.

La autoevaluación es un factor importante en la promoción del aprendizaje autorregulado, pues es una actividad que permite al aprendiz reflexionar sobre sus avances y logros en la tarea, al activar el monitoreo, lo que genera juicios, insumo fundamental para que el aprendiz pueda accionar el control metacognitivo que lo llevará a hacer ajustes durante la Fase III, de ejecución de la tarea,y/o cambios posteriores (Fase IV), que se aplicarán al momento de abordar futuras tareas (Careless, 2016; Panadero y Alonso, 2013; Panadero, Andrade et al. 2018; Panadero, Jonsson et al., 2017; Panadero et al., 2016; Paris y Paris, 2001; Paris y Winograd, 2003; Tai et al., 2018).

9. La eVAluACión del producto final de la taRea No debe SER SOLO SUMATIVA, SINO FORMATIVA

Para realizar una evaluación óptima de los estudiantes al final de la tarea, que apoye su proceso de autorregulación, se puede tener en cuenta:

1. La evaluación, además de dar una valoración cuantitativa de 1 a 5, debe proveer a los estu- diantes con valoraciones cualitativas, que resalten sus aciertos y desaciertos en la tarea. Estas valoraciones pueden formularse tomando en cuenta los criterios de evaluación y demandas de la tarea. Estos sirven de guía para evitar dar valoraciones sesgadas y fuera del contexto de la tarea acerca del desempeño de los estudiantes.

2. La evaluación debe tener una intención formativa, que permita a los estudiantes recibir información sobre los aspectos fuertes de su desempeño y aprendizaje en las actividades académicas y sobre los errores en los que incurren, mostrándolos como una oportunidad para aprender y optimizar el aprendizaje en otras tareas (Coll et al., 2012; Panadero, Andrade et al., 2018; Panadero et al., 2019). De esta forma, se ayudará al alumno a adoptar una postura reflexiva sobre su proceso de aprendizaje y se hará de la evaluación una actividad constructiva, alejándose del carácter amenazante que generalmente se le atribuye (Perry y VandeKamp, 2000). 
Las anteriores recomendaciones en relación con la evaluación son útiles para el aprendizaje autorregulado en la medida en que apoyan la reflexión de los estudiantes sobre su desempeño al final de la tarea (Fase IV: Adaptación metacognitiva). Las valoraciones externas que se den sobre el desempeño final de los estudiantes en la tarea contribuirán a que entiendan lo que lograron, lo que faltó por lograr y por qué. Esto contribuye a fortalecer sus conocimientos sobre el dominio de conocimiento de la tarea y sobre los métodos de estudio para abordar tareas, además de fortalecer aspectos motivacionales, como su autoeficacia para abordar tareas futuras en el dominio y el valor que les dan a las mismas (Garello y Rinaudo, 2013; Hawe y Dixon, 2017; Nicol, 2009; Panadero y Alonso, 2013; Panadero, Andrade et al., 2018; Panadero et al., 2019).

\section{Conclusiones}

Las pautas aquí presentadas se basan en los trabajos de investigadores en el tema, quienes en sus estudios han comprobado la efectividad de diferentes estrategias para mejorar las habilidades de autorregulación en los estudiantes en distintos niveles de educación. Por tanto, es necesario seguir generando más evidencia sobre la efectividad de esas estrategias en el contexto universitario; por ejemplo, Panadero, Andrade y Brookhart (2018) proponen que se debe seguir profundizando en los efectos de la evaluación formativa o centrada en el aprendizaje, durante el proceso de autorregulación del aprendizaje de los estudiantes, viendo específicamente qué componentes de este proceso son beneficiados por esa evaluación.

Asimismo, existen investigaciones que han probado que las estrategias de autorregulación que despliegan los estudiantes pueden variar según el dominio de conocimiento donde se encuentran las tareas (Greene et al., 2015) y según la experticia previa de los estudiantes en el mismo (Lajoie et al., 2013). Tomando en cuenta que en el ámbito univer- sitario se enseñan una gran variedad de contenidos en múltiples dominios y que los estudiantes pueden tener diferencias importantes en el conocimiento previo, sería importante: a) precisar si las pautas presentadas favorecen o no de igual forma el aprendizaje autorregulado en esos diferentes dominios y cuáles serían las formas de idóneas de promover la autorregulación según los dominios; y b) proponer una instrucción diferenciada (Akos et al., 2007) para promover el aprendizaje autorregulado, en la que esas pautas para el diseño y ejecución de tareas puedan ajustarse según el nivel de conocimientos previos de los estudiantes.

Por otra parte, las pautas presentadas pueden orientar la generación de talleres y programas psicoeducativos que se dirijan a profesores universitarios, con el fin de fortalecer el diseño y ejecución que estos hacen de tareas académicas, de modo que estas logren favorecer en los estudiantes la consolidación de habilidades de autorregulación: como la planeación, la persistencia, la elección y la ejecución de estrategias de aprendizaje idóneas para abordar las tareas, junto con la reflexión continua sobre su proceso de aprendizaje. Los talleres o programas que se realicen con profesores universitarios no solo deben centrarse en discutir y aplicar a la práctica de los profesores las pautas descritas, sino que también deben reflexionar sobre la importancia del aprendizaje autorregulado, sus diferentes fases y procesos y sus implicaciones en la forma de abordar la planeación, ejecución y reflexión de la enseñanza misma (Buttler et al. 2013; Kramarski, 2017).

La apropiación y aplicación de las pautas presentadas en la práctica pedagógica de los docentes estará influida por factores contextuales, como los tiempos que tienen para la preparación de clase y la atención a estudiantes, la cantidad de estudiantes que tengan en un curso y el volumen de contenidos que deben enseñar en los cursos (Daura, 2013). Estos factores pueden hacer difícil, por ejemplo, la generación de espacios de retroalimentación y au- 
toevaluación. Para esto es importante pensar en estrategias que permitan manejar los factores mencionados para mitigar su efecto negativo sobre las prácticas pedagógicas.

Estrategias como el uso de audiograbaciones para explicar las consignas y retroalimentar a los grupos de estudiantes cuando los cursos son numerosos (Broadbent et al., 2018) o trasladar la reflexión sobre la importancia de favorecer el aprendizaje autorregulado en la formación de profesionales a otros actores educativos, como directivos y administradores, para que la apuesta por su promoción sea un asunto institucional y se puedan tomar decisiones administrativas que faciliten a los profesores favorecer dicho proceso.
Finalmente, fomentar el aprendizaje autorregulado es para los profesores y los sistemas educativos tanto un medio como un fin (Häkkinen et al., 2017; Núñez et al., 2006). Un medio porque, favoreciendo el desarrollo de estrategias de autorregulación en los estudiantes, se tienen mayores posibilidades de incentivar aprendizajes de calidad en ellos; y un fin, pues para que los estudiantes logren ajustarse a los desafíos que plantea la universidad, la vida laboral y la sociedad de conocimiento, es necesario enseñar a los aprendices y futuros profesionales a: plantearse metas y dirigirse a ellas; usar estrategias pertinentes para lograrlas; seleccionar información adecuada; $y$ reflexionar sobre sus fortalezas y debilidades como aprendices, para que puedan seguir aprendiendo y usen esos aprendizajes en diferentes contextos.

\section{Referencias}

Abar, B. y Loken, E. (2010). Self-regulated learning and self-directed study in a pre-college sample. Learning and Individual Differences, 20(1), 25-29. https://doi.org/10.1016/j.lindif.2009.09.002

Adie, L., Van der Kleij, F.y Cumming, J. (2018). The development and application of coding frameworks to explore dialogic feedback interactions and self-regulated learning. British Educational Research Journal, 44(4), 704-723. https://doi.org/10.1002/berj.3463

Akos, P., Cockman, C. y Strickland, C. (2007). Differentiating classroom guidance. Professional School Counseling, 10(5), 455-463. https://doi.org/10.1177/2156759X0701000502

Ben-Eliyahu, A. y Bernacki, M. L. (2015). Addressing complexities in self-regulated learning: A focus on dynamic relations, contingencies, and contextual factors. Metacognition and Learning, 10, 1-13. https://doi. org/10.1007/s11409-015-9134-6

Binkley, M., Erstad, O., Herman, J., Raizen, S., Ripley, M., Miller-Ricci, M. y Rumble, M. (2012). Defining twenty-first century skills. En B. McGawy E. Care (eds), Assessment and teaching of $21^{\text {st }}$ century skills (pp 17-66). Springer. https://doi.org/10.1007/978-94-007-2324-5_2

Boer, H., Donker, A., Kostons, D. y Van der Werf, G. (2018). Long-term effects of metacognitive strategy instruction on student academic performance: A meta-analysis. Educational Research Review, 24, 98-115. https://doi. org/10.1016/j.edurev.2018.03.002 
Broadbent, J. y Poon, W. (2015). Self-regulated learning strategies \& academic achievement in online higher education learning environments: A systematic review. The Internet and Higher Education, 27, 1-13. http:// dx.doi.org/10.1016/j.iheduc.2015.04.007

Broadbent, J., Panadero, E.y Boud, D. (2018). Implementing summative assessment with a formative flavour: A case study in a large class. Assessment \& Evaluation in Higher Education, 43(2), 307-322. https://doi.org/10 $.1080 / 02602938.2017 .1343455$

Bruijn-Smolders, M., Timmers, C., Gawke, J., Schoonman, W. y Born, M. (2016). Effective selfregulatory processes in higher education: Research findings and future directions. A systematic review. Studies in Higher Education, 41(1), 139-158. https://doi.org/10.1080/03075079.2014.915302

Buttler, D., Schnellert, L. y Cartier, S. (2013). Layer of self and co-regulation: Teachers working collaboratively to support adolescent's self-regulated learning through reading. Education Research International, 2013, 1-19. http://dx.doi.org/10.1155/2013/845694

Capote, L., Rizo, N. y Bravo, G. (2017). La autorregulación del aprendizaje en estudiantes de la carrera de ingeniería industrial. Revista Universidad y Sociedad, 9(2), 44-52. http://scielo.sld.cu/scielo.php?script=sci_ abstract\&pid=S2218-36202017000200005\&lng=es\&nrm=iso

Careless, D. (2013). Trust and its role in facilitating dialogic feedback. En D. Boud y E. Molloy (eds.), Feedback in higher and professional education: Understanding it and doing it well (pp. 90-103). Routledge.

Careless, D. (2016). Feedback as dialogue. En M. Peters (ed.), Encyclopedia of Educational Philosophy and Theory (pp. 1-6).Springer. https://doi.org/10.1007/978-981-287-532-7_389-1

Careless, D. y Boud, D. (2018). The development of student feedback literacy: Enabling uptake of feedback. Assesment \& Evaluation in Higuer Education, 43(8), 1315-1325. https://doi.org/10.1080/02602938.2018.1463354

Chocarro, E., González-Torres, M. y Sobrino, A. (2007). Nuevas orientaciones en la formación del profesorado para una enseñanza centrada en la promoción del aprendizaje autorregulado. Estudios sobre Educacion, 12, 8198. https://revistas.unav.edu/index.php/estudios-sobre-educacion/article/view/24318

Coll, C., Mauri, T.y Rochera, J. (2012). La práctica de evaluación como contexto para aprender a ser un aprendiz competente. Profesorado. Revista de Curriculum y formación del Profesorado, 16(1), 50-59. http://www.ugr. es/ recfpro/rev161ART4.pdf

Daura, F. (2011). Las estrategias docentes al servicio del desarrollo del aprendizaje autorregulado. Estudios Pedagógicos, 37(2), 77-88. http://dx.doi.org/10.4067/S0718-07052011000200004

Daura, F. (2013). El contexto como factor del aprendizaje autorregulado en la educación superior. Educación y Educadores, 16(1), 109-125. https://doi.org/10.5294/edu.2013.16.1.7

Daura, F. (2015). Aprendizaje autorregulado y rendimiento académico en estudiantes del ciclo clínico de la carrera de Medicina. Revista Electrónica de Investigación Educativa, 17(3), 29-45. https://redie.uabc.mx/redie/ article/view/408 
De la Fuente, J., Berbén, A. y Zapata, L. (2013). How regulatory teaching impacts university student's perceptions of the teaching-learning process: The roll of teacher training. Journal for the Study of Education and Development, 36(3), 375-385. https://doi.org/10.1174/021037013807533016

Dent, A. y Koenka, A. (2016). The relation between self-regulated learning and academic achievement across childhood and adolescence: A meta-analysis. Educational Psychology Review, 28(3), 425-474. https://doi. org/10.1007/s10648-015-9320-8

Dignath, C. y Van der Werf, G. (2012). What teachers think about self-regulated learning: Investigating teacher beliefs and teacher behavior of enhancing students' self-regulation. Education Research International, 2012, 1-10. https://doi.org/10.1155/2012/741713

Dignath-van Ewijk, C., Dickhäuser, O. y Büttner, G. (2013). Assessing how teachers enhance self-regulated learning -a multi-perspective approach. Journal of Cognitive Education and Psychology, Special Issue on SelfRegulated Learning, 21,338-358. https://doi.org/10.1891/1945-8959.12.3.338

Dignath, C. (2016). Which components of teacher competence determine whether teachers enhance self-regulated learning? Frontline Learning Research, 4(5), 83-105. http://dx.doi.org/10.14786/flr.v4i5.247

Dignath, C. y Büttner, G. (2018). Teachers' direct and indirect promotion of self-regulated learning in primary and secondary school mathematics classes -insights from video-based classroom observations and teacher interviews. Metacognition and Learning, 13(2), 127-157. https://doi.org/10.1007/s11409-018-9181-X

Donker, A. S., De Boer, H., Kostons, D., Van Ewijk, C. D. y Van der Werf, M. P. (2014). Effectiveness of learning strategy instruction on academic performance: A meta-analysis. Educational Research Review, 11, 1-26. https://doi. org/10.1016/j.edurev.2013.11.002

Fraile, J., Pardo, R. y Panadero, E. (2017a). ¿Cómo emplear las rúbricas para implementar una verdadera evaluación formativa? Revista Complutense de Educación, 28(4), 1321-1334. https://doi.org/10.5209/RCED.51915

Fraile, J., Panadero, E. y Pardo, R. (2017b). Co-creating rubrics: The effects on self-regulated learning, self-efficacy and performance of establishing assessment criteria with students. Studies in Educational Evaluation, 53, 69-76. https://doi.org/10.1016/j.stueduc.2017.03.003

Garello, V.y Rinaudo, M. (2012). Características de las tareas académicas que favorecen el aprendizaje autorregulado y la cognición distribuida en estudiantes universitarios. Revista de Docencia Universitaria, 10(3), 415-440. https://doi.org/10.4995/redu.2012.6030

Garello, M. y Rinaudo, M. (2013). Autorregulación del aprendizaje, feedback y transferencia de conocimiento. Investigación de diseño con estudiantes universitarios. Revista Electrónica de Investigación Educativa, 15(2), 131-147. http://www.scielo.org.mx/pdf/redie/v15n2/v15n2a9.pdf

Greene, J. y Azevedo, R. (2007). A theoretical review of Winne and Hadwin's model of self-regulated learning. Review of Educational Research, 77(3), 334-372. https://doi.org/10.3102/003465430303953 
Greene, J., Hutchinson, L., Costa, L-J. y Crompton, H. (2012). Investigating how college students' task definitions and plans relate to self-regulated learning processing and understanding of a complex science topic. Contemporary Educational Psychology, 37, 307-320. https://doi.org/10.1016/j.cedpsy ch.2012.02.002

Greene, J., Mason, C., Jackson, W., Caprino, A., Oswald, C. y McVea, M. (2015). Domain-specificity of self-regulated learning processing in science and history. Contemporary Educational Psychology, 42, 111-128. https://doi. org/10.1016/j.cedpsy ch.2015.06.001

Gynnild, V., Holstad, A. y Myrhaug, D (2008). Identifying and prompting self-regulated learning in higher education: Roles and responsibilities of students tutor. Mentoring y Tutoring: Partnership y Learning, 16(2), 147-161. https://doi.org/10.1080/13611260801916317

Hadwin, A., Wozney, L.y Pontin, O. (2005). Scaffolding the appropriation of self-regulatory activity: A socio-cultural analysis of changes in teacher-student discourse about a graduate portfolio. Instructional Science, 33 , 413-450. https://doi.org/10.1007/s11251-005-1274-7

Hadwin, A., Järvelä, S. y Miller, M. (2011). Self-regulated, co-regulated, and socially shared regulation of learning. En B. Zimmerman, y D. Schunk (eds.), Handbook of Self-Regulation of Learning and Performance (pp. 65-84). Routledge.

Hadwin, A. (2013). Response to Vasallo's claims from historically situated view of self-regulated learning as adaptation in the face of challenge. New Ideas in Psychology, 31(3), 1-4. https://doi.org/10.1016/j.newideapsy ch.2012.05.001

Hadwin, A., Järevelä, S. y Miller, M. (2017). Self-regulation, co-regulation, and shared regulation in collaborative learning environments. P. Alexander, D. Schunk y J. Greene (eds.), Handbook of self-regulation of learning and performance (pp. 83-106). Routledge.

Häkkinen, P., Järvelä, S., Mäkitalo-Siegl, A., Näykki, P. y Valtonen, T. (2017). Preparing teacher-students for twentyfirst century learning practices (PREP-21): A framework for enhancing collaborative problem solving and learning skills. Teacher and Teaching, 23(1), 25-41. https://doi.org/10.1080/13540602.2016.1203772

Hattie, J. y Temperley, H. (2007). The power of feedback. Review of Educational Research, 77(1). https://doi. org/10.3102/003465430298487

Hattie, J. y Gan, M. (2011). Instruction based on feedback. En R. E. Mayer y P. A. Alexander (eds.), Handbook of Research on Learning and Instruction (pp. 249-271). Routledge.

Hawe, E.y Dixon, H. (2017). Assessment for learning: a catalyst for student self-regulation. Assessment \& Evaluation in Higher Education, 42(8), 1181-1192. https://doi.org/10.1080/02602938.2016.1236360

Hoops, L., Yu, S., Wang, O. y Hollyer, V. (2016). Investigating postsecondary self-regulated learning instructional practices: The development of the self-regulated learning observation protocol. International Journal of Teaching and Learning in Higher Education, 28(1), 75-93. https://eric.ed.gov/?id=EJ1106342

Kitsantas, A. (2002). Test preparation and test performance: A self-regulatory analysis. The Journal of Experimental Education, 70(2), 101-113. https://doi.org/10.1080/00220970209599501 
Kistner, S., Rakoczy, K., Otto, B.y Klieme, E. (2015). Teaching learning strategies: The role of instructional context and teacher beliefs. Journal for Educational Research Online, 7(1), 176-197.

Koivuniemi, M., Panadero, E., Malmberg, J., y Järvelä, S. (2017). Desafíos de aprendizaje y habilidades de autorregulación en distintas situaciones de aprendizaje en estudiantes de educación superior. Infancia y Aprendizaje, 4O(1), 19-55. https://doi.org/10.1080/02103702.2016.1272874

Kramarski, B. y Michalsky, T. (2010). Preparing preservice teachers for self-regulated learning in the context of technological pedagogical content knowledge. Learning and Instruction, 20, 434-447. https://doi. org/10.1016/j.learninstruc.2009.05.003

Kramarski, B., Desoete, A., Bannert, M., Narciss, S. y Perry, N. (2013). New perspectives on integrating self-regulated learning at school. Education Research International, 2013, 1-4. http://dx.doi.org/10.1155/2013/498214

Kramarski, B. y Kohen, Z. (2016). Prompting preservice teachers? Dual self-regulation roles as learners and as teachers: Effects of generic vs specific prompts. Metacognition and Learning, 12, 157-191. https://doi. org/10.1007/s11409-016-9164-8

Kramarski, B. (2017). Teachers as agents in promoting students' SRL and performance Applications for Teachers' Dual-Role Training Program. En P. Alexander, D. Schunk, y J. Greene (eds.), Handbook of Self-Regulation of Learning and Performance Routledge. Routledge.

Lajoie, S. (2008). Metacognition, self-regulation, and self-regulated learning: A rose by any other name? Educational Psychology Review, 20(4), 469-475. https://doi.org/10.1007/s10648-008-9088-1

Lajoie, S., Naismith, L., Poitras, E., Hong, Y., Cruz-Panesso, I., Ranellucci, J.y Wiseman, J. (2013). Technology-rich tools to support self-regulated learning and performance in medicine. En R. Azevedo y V. Aleven (eds.), International Handbook of Metacognition and Learning Technologies (pp. 229-242). Springer.

Lawanto, O. y Febrian, A. (2017, feb.). Student's self-regulation in senior capstone design proyects: A case study of two proyect teams. Frontiers in Education Conference (FIE). https://doi.org/10.1109/FIE.2016.7757481

León, J., Núñez, J.y Liew, J. (2015). Self-determination and STEM Education: effects of autonomy, motivation and self -regulated learning on high school math achievement. Learning and Individual Differences, 43, 156163. https://doi.org/10.1016/j.lindif.2015.08.017

Ley, K.y Young, D. (2001). Instructional principles for self-regulation. Educational Technology Research and Development, 49(2), 93-103. https://doi.org/10.1007/BF02504930

Lodewyk, K., Winne, P. y Jamieson-Noel, D. (2009). Implication of task structure on self-regulated learning and achievement. Educational Psychology, 29, 1-25. https://doi.org/10.1080/01443410802447023

McCardle, L. y Hadwin, A. (2015). Using multiple, contextualized data sources to measure learners' perceptions of their self-regulated learning. Metacognition and Learning, 10, 43-75. https://doi.org/10.1007/s11409-014-9132-0 
McMahon, M. y Oliver, R. (2001). Promoting self-regulated learning in an on-line environment. En C. Montgomerie y J.Viteli (eds.), Proceedings of World Conference on Educational Multimedia, Hypermedia and Telecommunications 2001 (pp. 1299-1305). AACE. https://ro.ecu.edu.au/cgi/viewcontent.cgi?referer=https://www. google.com $/$ \&httpsredir $=1 \&$ article $=5815 \&$ context $=$ ecuworks

Ministerio de Educación Nacional (2016). Plan Nacional Decenal de Educación 2016-2026. El camino hacia la equidad. http://www.plandecenal.edu.co/cms/images/PLAN\%2ONACIONAL\%2ODECENAL\%2ODE\%2O EDUCACION\%2O2DA\%2OEDICION_271117.pdf

Montes, J., Ayala, I. y Atencio, F. (2005). Preparación para exámenes y aprendizaje autorregulado con estudiantes universitarios. Pensamiento Psicológico, 1(6), 57-71. https://revistas.javerianacali.edu.co/index.php/pensamientopsicologico/article/view/20

Moos, D. C.y Ringdal, A. (2012). Self-regulated learning in the classroom: A literature review on the teacher's role. Education Research International, 2012, 1-15. https://doi.org/10.1155/2012/423284

Nelson, K., Shell, D., Husman, J., Fishman, E. y Soh, L-K. (2015). Motivational and self-regulated learning profiles of students taking a foundational engineering course. Journal of Engineering Education, 104(1), 74-100. https://doi.org/10.1002/jee.20066

Nicol, D. y MacFarlane-Dick, D. (2006). Formative assessment and self-regulated learning: A model of seven principles of good feedback practice. Studies in Higher Education, 31(2), 199-218. http://www.psy.gla. ac.uk/ steve/rap/docs/nicol.dmd.pdf

Nicol, D. (2009). Assessment for learner self-regulation: Enhancing achievement in the first year using learning technologies. Assessment and Evaluation in Higher Education, 34(3), 335-352. https://doi. org/10.1080/02602930802255139

Núñez, J., Solano, P., González-Pineda, J.y Rosario, P. (2006). El aprendizaje autorregulado como medio y meta de la educación. Papeles del Psicólogo, 27(3), 139-146. https://www.redaly c.org/pdf/778/77827303.pdf

Orozco, M., Ochoa-Angrino, S. y Sánchez, H. (2002). Prácticas culturales para la educación en la niñez. Itinerario para recuperar y significar prácticas culturales desde la perspectiva del desarrollo. Centro de Investigaciones y Estudios Avanzados en Psicología, Cognición y Cultura, Universidad del Valle.

Panadero, A. y Alonso-Tapia, A. (2013). Self-assessment: Theoretical and practical connotations. When it happens, how is it acquired and what to do to develop it in our students. Electronic Journal of Research in Educational Psychology, 11(2), 551-576. http://dx.doi.org/10.14204/ejrep.30.12200

Panadero, E., Alonso-Tapia, J.y Reche, E. (2013). Rubrics vs self-assessment scripts effect on self-regulation, performance and self-efficacy in preservice teachers. Studies in Educational Evaluation, 39, 125-132. https://doi. org/10.1016/j.stueduc.2013.04.001

Panadero, A. y Jonsson, A. (2013). The use of scoring rubrics for formative assessment purposes revisited: A review. Educational Research Review, 9, 129-144. https://doi.org/10.1016/j.edurev.2013.01.002 
Panadero, A., Alonso-Tapia, A. y Huertas, J. (2014). Rubrics vs. self-assessment scripts: effects on first year university students' self-regulation and performance. Journal for the Study of Education and Development, 37(1), 149-183. https://doi.org/10.1080/02103702.2014.881655

Panadero, E., Jonsson, A. y Strijbos, J. W. (2016). Scaffolding self-regulated learning through self-assessment and peer assessment: Guidelines for classroom implementation. En D. Laveault y L. Allal (eds.), Assessment for Learning: Meeting the Challenge of Implementation. https://doi.org/10.1007/978-3-319-39211-0_18

Panadero, E., Jonsson, A. y Botella, J. (2017). Effects of self-assessment on selfregulated learning and self-efficacy: Four meta-analyses. Educational Research Review, 22, 74-98. https://doi.org/10.1016/j.edurev.2017.08.004

Panadero, E., Andrade, H. y Brookhart, S. (2018). Fusing self-regulated learning and formative assessment: a roadmap of where we are, how we got here, and where are we going. The Australian Educational Research, 45(1), 13-31. https://doi.org/10.1007/s13384-018-0258-y

Panadero, E., Jonsson, A. y Alqassab, M. (2018). Providing formative peer feedback: What do we know? En A. A. Lipnevich y J. K. Smith (eds.), The Cambridge Handbook of Instructional Feedback. Cambridge University Press.

Panadero, E., Broadbent, J., Boud, D. y Lodge, J. (2019). Using formative assessment to influence self- and coregulated learning: The role of evaluative judgement. European Journal of Psychology of Education, 34(3), 535-557. https://doi.org/10.1007/s10212-018-0407-8

Paris, S. G.y Paris, A. H. (2001). Classroom applications of research on self-regulated learning. Educational Psychologist, 36(2), 89-101. https://doi.org/10.1207/S15326985EP3602_4

Paris, S.y Winograd, P. (2003). The role of self-regulated learning in contextual teaching: Principals and practices for teacher preparation. https://files.eric.ed.gov/fulltext/ED479905.pdf

Perry, N. (1998). Young children's self-regulated learning and contexts that support it. Journal of Educational Psychology, 90(4), 715-729. http://dx.doi.org/10.1037/0022-0663.90.4.715

Perry, N. y VandeKamp, K. (2000). Creating classroom contexts that support young children's development of self-regulated learning. International Journal of Educational Research, 33, 821-843. https://doi.org/10.1016/ So883-0355(00)00052-5

Perry, N., VandeKamp, K., Mercer, L. y Nordby, C. (2002). Investigating teacher-student interactions that foster self-regulated learning. Educational Psychologist, 37 (1), 5-15. https://doi.org/10.1207/S15326985EP3701_2

Perry, N., Philips, L. y Dowler, J. (2004). Examining features of tasks and their potential to promote self-regulated learning. Teacher College Record, 106(9), 1854-1878. https://doi.org/10.1111/j.1467-9620.2004.00408.x

Perry, N., Phillips, L.y Hutchinson, L. (2006). Mentoring student teachers to support self-regulated learning. The Elementary School Journal, 106(3), 237-254. https://doi.org/10.1086/501485 
Perry, N., Hutchinson, L. y Thauberger, C. (2008). Talking about teaching self-regulated learning: Scaffolding student teachers' development and use of practices that promote self-regulated learning. International Journal of Educational Research, 47(2), 97-108. https://doi.org/10.1016/j.jjer.2007.11.010

Perry, N. y Rahim, A. (2011). Studying self-regulated learning in classrooms. En B. J. Zimmerman y D. H. Schunk (eds.), Handbookof Self-regulation of Learning and Performance (pp. 122-136). Routledge

Perry, N., Hutchinson, L., Yee, N. y Määttä, E. M. (2018). Advances in understanding young children's self-regulation of learning. En P. Alexander., D. Schunk y J. Greene (eds.) Handbook of Self-Regulation of Learning and Performance (pp.457-472). Routledge.

Peeters, J., De Backer, F., Romero, V., Kindekens, A., Buffel, T. y Loembaerts, K. (2014). The role of teachers' self-regulatory capacities in the implementation of self-regulated learning practices. Procedia-Social and Behavioral Sciences, 116, 1963-1970. https://doi.org/10.1016/j.sbspro.2014.01.504

Puntel, F.y Menna, M. (2018). Teaching activities that develop learning self-regulation. Educaoy Realidade, 43(2), 495-511. http://dx.doi.org/10.1590/2175-623665212

Schunk, D. y Greene, J. (2017). Historical, contemporary and future perspectives on self- regulated learning and performance. En P. Alexander., D. Schunk y J. Greene (eds.), Handbook of Self-Regulation of Learning and Performance (pp.1-15). Routledge.

Shell, D. y Soh, L. (2013). Profiles of motivated self-regulation in college computer science courses: Differences in major versus required no-major courses. Journal of Science Education and Technology, 22(6), 889-913. https://doi.org/10.1007/s10956-013-9437-9

Rojas, T. y Valencia, M. (en revisión). Autorregulación de la motivación de estudiantes universitarios y su relación con aspectos situacionales en clases de matemáticas. Manuscrito enviado para publicación.

Rosario, P., Pereira, A., Högemann, J., Nunes, A., Figuereido, M., Núñez, J., Fuente, S. y Gaeta, M. (2014). Autorregulación del aprendizaje: Una revisión sistemática en revista de base Scielo. Universitas Pshychologica, 13(2), 871-797. https://revistas.javeriana.edu.co/index.php/revPsy cho/article/view/4487

Severin, E. (2017). Un nuevo paradigma educativo. Educación y Ciudad, 32, 75-82. https://dialnet.unirioja.es/servlet/articulo?codigo $=6213563$

Tai, J., Ajjwai, R., Boud, D., Dawson, P.y Panadero, E. (2018). Developing evaluative judgement: Enabling students to make decisions about the quality of work. Higher Education, 76(3), 467-481. https://doi.org/10.1007/ s10734-017-0220-3

To, J. y Panadero, E. (2019). Peer assessment effects on the self-assessment process of first -year undergraduates. Assessment \& Evaluation in Higher Education, 44(6), 920-932. https://doi.org/10.1080/02602938.2018.1548559

Torrano, F., Fuentes, J. y Soria, M. (2017). Aprendizaje autorregulado: estado de la cuestión y retos psicopedagógicos. Perfiles Educativos, 39(156), 160-173. https://doi.org/10.22201/iisue.24486167e.2017.156.58290 
Tuysuzoglu, B. B.y Greene, J. (2015). Investigation of the role of contingent metacognitive behavior in self-regulated learning. Metacogntition and Learning, 10(1), 43-75. https://doi.org/10.1007/s11409-014-9126-y

Valencia, M., Duarte, J. y Caicedo, A. M. (2013). Aprendizaje autorregulado, metas académicas y rendimiento en evaluaciones de estudiantes universitarios. Pensamiento Psicológico, 11(2), 53-70. https://revistas.javerianacali.edu.co/index.php/pensamientopsicologico/article/view/428

Valencia, M. y Caicedo, A. (2017). Diseño de tareas apoyadas en TIC para promover aprendizaje autorregulado. Pensamiento Psicológico, 15(2), 15-28. https://revistas.javerianacali.edu.co/index.php/pensamientopsicologico/article/view/1586

Vrieling, E., Bastiens, T. y Stijnen, S. (2012). Effects of increased self-regulated learning opportunities on student teachers' metacognitive and motivational development. International Journal of Educational Research, 53, 251-253. https://doi.org/10.1016/j.ijer.2012.03.014

Wang, J., Liu, W. y Ryan, R. (2016). Can being autonomy-supportive in teaching improve students' self-regulation and performance? En W. Liu, J. Wang y R. Ryan, Building autonomous learners (pp. 227-243). Springer. https://doi.org/10.1007/978-981-287-630-0_12

William, D. (2014, april). Formative assessment and contingency in the regulation of learning processes. [Symposium] Toward a Theory of Classroom Assessment as the Regulation of Learning, American Educational Research Association, Philadelphia, PA.

Winne, P.y Buttler, D. (1995). Feedback and self-regulated learning: A Theoretical Synthesis. Review of Educational Research, 65(3), 245-281. https://doi.org/10.2307/1170684

Winne, P. y Hadwin, A. (1998). Studying as self-regulated learning. En D. Hacker, J. Dunlosky y A. Graesser (eds.), Metacognition into Theory and Practice (pp. 277-304). Lawrence Erlbaum.

Winne, P. y Jamieson-Noel, D. L. (2002). Self-regulating studying by objectives for learning: Students' reports compared to a model. Contemporary Educational Psychology, 28, 259-276. http://dx.doi.org/10.1016/So361476X(02)00041-3

Winne, P. y Hadwin, A. (2008). The wave of motivation and self-regulated learning. En D. H. Schunk y B. J. Zimmerman (eds.), Motivation and self-regulated learning: Theory, research, and applications (pp. 297-314). Lawrence Erlbaum.

Winne, P. (2011). A cognitive and metacognitive analysis of self-regulated learning. En B.Zimmerman y D. Schunk (eds.), Handbook of Self-Regulation of Learning and Performance (pp.15-32). Routledge.

Winne, P. (2017). Cognition and metacognition within self-regulated learning. En P. Alexander., D. Schunk y J. Greene (eds.), Handbook of Self-Regulation of Learning and Performance (pp. 36-48). Routledge.

Winne, P. (2018). Theorizing and researching levels of processing in self-regulated learning. British Journal of Educational Psychology, 88(1), 9-20. https://doi.org/10.1111/bjep.12173 
ISSN $0123-1294$ | e-ISSN 2027-5358 | Educ.Educ. Vol. 23. No. 2 | Mayo-julio de 2020 | pp. 267-290

Universidad de La Sabana | Facultad de Educación

Zimmerman, B. (2000). Attainment of self-regulation: A social cognitive perspective. En M. Boekaerts, P. R. Pintrich, y M. Zeidner (eds.), Handbook of self-regulation (pp.13-39). Academic Press.

Zimmerman, B. (2002). Becoming a self-regulated learner: An overview. Theory into Practice, 41(2), 64-70. https:// doi.org/10.1207/s15430421tip4102_2

Zimmerman, B.y Moylan, A. (2009). Self-regulation:When metacognition and motivation intersect. En D. J. Hacker, J. Dunlosky, y A. C. Graesser (eds.), Handbook of Metacognition in Education (pp. 299-315). RoutledgeTaylor \& Francis.

Zimmerman, B. (2011). Motivational sources and outcomes of self-regulated learning and performance. En B. Zimmerman y D. Schunk (eds.), Handbook of Self-Regulation of Learning and Performance (pp. 49-64). Routledge.

Zimmerman, B. (2013). From cognitive modeling to self-regulation: a social cognitive career path. Educational Psychology, 48(3), 135-147. https://doi.org/10.1080/00461520.2013.794676 